\title{
VOLATILIDAD DE PRECIOS INTERNACIONALES RECIBIDOS POR LOS PRODUCTORES DE KIWIS Y MANZANAS FRESCAS CHILENAS ${ }^{1}$
}

\author{
GERMÁN LOBOS ANDRADE² \& JOSÉ LUIZ PETRI ${ }^{3}$
}

RESUMEN-El objetivo de este trabajo fue analizar el comportamiento de los precios medios FOB y el de los precios medios recibidos por los productores de kiwis y manzanas frescas chilenas, usando datos mensuales del periodo enero 1998 a diciembre 2005. Los valores fueron expresados en moneda de diciembre de 2005 usando como deflactor el WPI de EE.UU. Las series de precios medios recibidos por los productores se estimaron indirectamente restando a los precios medios FOB las comisiones y tarifas de exportación. Como medida de volatilidad se usó la desviación estándar de los retornos (variación de precios) continuos de cada serie. Se utilizó el método del promedio geométrico móvil para estimar patrones de estacionalidad ajustada de los precios recibidos por los productores de kiwis y manzanas frescas chilenas. Se observó una mayor volatilidad de los retornos en kiwis $(47,5 \%)$ que en manzanas (17,3\%). Los resultados mostraron: a) una menor estacionalidad de precios para kiwis que manzanas; b) una estabilidad de precios en marzo y desde julio a noviembre para kiwis, y desde febrero a junio y desde agosto a diciembre para manzanas; c) un valor máximo en diciembre y más bajo en junio para kiwis, un valor máximo en julio y más bajo en enero para manzanas.

Términos para indexación: Estacionalidad ajustada, promedio móvil, estabilidad, riesgo.

\section{VOLATILITY OF INTERNATIONAL PRICES RECEIVED BY CHILEAN FRESH KIWI AND APPLE FARMERS}

\begin{abstract}
The purpose of this study is to analyze the behavior of FOB average prices and the average prices received by fresh kiwi and apple producers, using monthly figures for the period spanning from January 1998 to December 2005 . The values were expressed in December 2005 currency rates using the U.S.A. WPI (Wholesale Price Index) as a deflator. The series of average prices received by the producers were estimated indirectly by subtracting the commissions and export tariffs from the FOB average prices. As a measure of volatility, the standard deviation of the continuous returns (prices variation) of each series was used. The patterns of seasonally adjusted price fluctuations, received by Chilean fresh kiwi and apple farmers, were estimated through the geometric mobile average method. A greater volatility in the returns was observed in kiwi $(47,5 \%)$ than in apples $(17,3 \%)$. The results showed: a) lower price seasonality for kiwi than for apples; b) price stability in march and from July through November, for kiwi, and from February to June and from august through December, for apples; c) peak value in December and lowest in June, for kiwi; peak value in July and lowest in January, for apples.
\end{abstract}

Index terms: Adjusted seasonally, mobile average, stability, risk.

\section{INTRODUCCIÓN}

Según cifras de la FAO (2006), la producción mundial de kiwis durante la temporada 2005/2006 (julio-junio) se estima en aproximadamente 1,1 millones de TM; esta cifra fue superior en un $7 \%$ con respecto a la temporada 2004/2005. Por otra parte, la producción mundial de manzanas durante la temporada 2005/ 2006 (julio-junio) se estima en aproximadamente 63 millones de $\mathrm{TM}$, con un incremento de sólo un $0,5 \%$ con respecto a la temporada anterior. En términos de volumen la producción de Chile representa un $13 \%$ (kiwis) y $2 \%$ (manzanas) de la producción mundial estimada.

Los principales países productores de kiwis frescos son Italia, Nueva Zelanda, Chile, Francia y Grecia. En la producción de manzanas frescas, China es el principal productor mundial (30\%), seguido de EE.UU., Turquía, Polonia, Italia, Francia y
Rusia; en el Hemisferio Sur, los productores más importantes son Chile, Sudáfrica y Nueva Zelanda. En Chile las exportaciones de kiwis frescos superan el $70 \%$ de la producción doméstica y el $55 \%$ en el caso de las manzanas frescas (USDA, 2006).

De acuerdo a ODEPA (2006), en Chile la superficie plantada con kiwis se estima en 6.640 ha. La superficie plantada con manzanos se estima en 36.095 ha, de las cuales un 29.455 ha corresponde a manzano rojo y 6.640 ha a manzano verde. Las principales variedades de manzanas comercializadas en los mercados internacionales son Royal Gala, Granny Smith y Richard Delicius; en menor medida se comercializan Fuji, Red Starking y Braebum. El volumen de exportaciones de kiwis frescos chilenos pasó de 102,5 a 131,5 TM entre el año 2000 y 2005, y de manzanas frescas aumentó de 387,7 a 639,4 en el mismo periodo. Según Emden (2004), en Chile la cosecha de manzanas Gala y Royal Gala se inicia durante principios de febrero y se mantiene hasta fines de mayo. Según el autor, las variedades de manzanas con mayor capacidad de

${ }^{1}$ (Trabalho 053-07) Recibido el : 01-03-2007.Aceptado para publicación el: 06-09-2007. Apoyo financiero: Fondo de Desarrollo Académico, FACE, Universidad de Talca.

${ }^{2}$ Magíster en Economía Agraria (PUC), Universidad de Talca, C.P. 346-5548, Tel.: 71-200330, Talca, Chile, E-mail: globos@utalca.cl

${ }^{3}$ M.Sc., Pesquisador da EPAGRI - Caçador SC e Professor da UNC (Universidade do Contestado), Fone 49-35612000, Brasil, E-mail: petri@epagri.rct-sc.br 
almacenamiento son Fuji, Jonagold, Granny y Starkimson Delicious, que se exportan hasta fines de noviembre, mientras que las Galas son de corta duración.

Existe amplia evidencia que indica que la volatilidad de precios es importante en los commodities agrícolas (Hudson \& Coble, 1999; Goodwin \& Schnepf, 2000; Buguk et al., 2003). Sekhar (2003b) sugiere que los precios de los productos agrícolas presentan una mayor variabilidad que los precios de los productos no-agrícolas. Además, diversas investigaciones (Starleaf, 1982; González et al., 2002; Sekhar, 2003a, 2003b) reportan que la alta volatilidad de los precios agrícolas puede ser atribuida a: (1) las bajas elasticidades precio-propio e ingreso de la demanda por productos agrícolas, (2) la inestabilidad inherente a la producción agrícola derivada de efectos externos no controlables por los productores, tales como las condiciones climáticas, (3) la naturaleza del proceso de producción agrícola, donde las decisiones de producción deben tomarse en un contexto de riesgo e incertidumbre respecto de las condiciones que prevalecerán en los mercados donde los productos son comercializados. El argumento (1) queda de manifiesto al observar que la mayoría de los trabajos reportados por la literatura concluyen que la demanda por fruta fresca es inelástica con respecto al precio-propio y que la elasticidad ingreso es positiva pero menor que 1 (Lee, 1994; Duval \& Biere, 2002; Agbola, 2003; Stewart et al., 2003). Para el caso de las manzanas frescas exportadas por Chile, similar conclusión se observa en los trabajos realizados por Cerda et al. (2004) y Lobos et al. (2005).

En el caso de Brasil, Morgado et al. (2004) calcularon índices de estacionalidad de los precios medios recibidos por los productores de piñas en Río de Janeiro, en base a datos mensuales del periodo enero 1995 a diciembre 2001. Los autores concluyeron que los precios medios presentan cierta estabilidad desde enero a octubre, mientras que los valores más bajos se observaron en los meses de noviembre y diciembre, época en que se concentra la producción. Lobos \& Muñoz (2005) estimaron índices de estacionalidad de los precios medios recibidos por los productores de manzanas frescas chilenas, usando datos mensuales del periodo enero 1990 a septiembre 2005. Los autores concluyeron que existe una estabilidad de precios para junio y desde septiembre a diciembre, con un valor máximo en julio y con los precios más bajos en abril y mayo. Troncoso \& Lobos (2004) analizaron la estabilidad de los precios y márgenes de comercialización para una canasta de frutas y hortalizas en Chile, utilizando datos mensuales del periodo enero 1993 a diciembre 2002. La principal conclusión sugiere que la componente estacional es la más relevante en todas las series analizadas.

El objetivo de este trabajo fue analizar el comportamiento de los precios medios FOB y el de los precios medios recibidos por los productores de kiwis y manzanos.

\section{MATERIALES Y MÉTODOS}

En este trabajo se analizó el comportamiento de los precios medios FOB de kiwis y manzanas frescas chilenas, medidos en US $\$ \mathrm{~kg}^{-1}$, usando datos mensuales del periodo enero 1998 a diciembre
2005, los cuales se obtuvieron de los registros históricos de ODEPA (2004). Los valores fueron expresados en moneda de diciembre de 2005 usando como deflactor el WPI (Wholesale Price Index) de EE.UU. Las series de precios medios FOB permitieron obtener los precios medios recibidos por los productores, los cuales se estimaron indirectamente restando a los primeros las comisiones y tarifas de exportación.

Los precios medios recibidos por los productores se obtuvieron utilizando el procedimiento indirecto de cálculo de su valor propuesto por Lobos \& Muñoz (2005). Este procedimiento consiste en descontar a los precios medios FOB los valores pagados por los productores a las empresas exportadoras, los cuales incluyen la comisión de exportación y las tarifas por servicios. Se utilizó la expresión

$$
P_{i j}^{P R O}=P_{i j}^{F O B}-c P_{i j}^{F O B}-\sum_{t=1}^{n} T_{t} \text {, donde } P^{P R O} \text { es el precio }
$$

medio recibido por los productores, los subíndices $i, j$ corresponden al año y mes, respectivamente, $c$ es la comisión porcentual de exportación, $T$ son las tarifas cobradas por las exportadoras, y el subíndice $t$ se refiere al tipo de tarifa.

Como medida de volatilidad se usó la desviación estándar de los retornos (variación de precios) continuos de cada serie; el procedimiento anterior requiere que cada una de las series de precios medios FOB y estimados a productor sea estacionaria, es decir, que no contenga crecimiento o declinación (según Hanke \& Reitsch, 1997, una serie estacionaria es aquella cuya media y varianza no cambian a través del tiempo). Dado que las series resultaron no estacionarias, se eliminó la tendencia a través del método de diferenciación calculando el retorno de los precios como $\left(\mathrm{P}_{\mathrm{t}} / \mathrm{P}_{\mathrm{t}-1}\right)$, donde $\mathrm{P}_{\mathrm{t}}$ es el precio en el mes " $\mathrm{t}$ " $\mathrm{y} \mathrm{P}_{\mathrm{t}-1}$ es el precio en el mes " $\mathrm{t}-1$ ". Posteriormente se calculó la desviación estándar (s) del retorno compuesto continuo como $\ln \left(\mathrm{P}_{t} / \mathrm{P}_{\mathrm{t}-\mathrm{1}}\right)$ para todo el periodo considerado, donde s es la medida de volatilidad estimada.

Para comparar la variabilidad de los precios medios FOB y estimados a productor de cada serie se calculó el coeficiente de variación $(\mathrm{CV})$ como la desviación estándar entre el promedio de dichos precios para todo el periodo considerado. El valor del CV permitió conocer la variabilidad de los precios medios con respecto al promedio de dichos precios, tanto para kiwis como manzanas.

Se aplicó el método de promedios geométricos móviles para determinar los patrones de variación estacional y un intervalo de dispersión de índices estacionales de los precios medios recibidos por los productores de kiwis y manzanas (Mendenhall \& Reinmuth, 1981; Maddala, 1996; Hanke \& Reitsch, 1997). Según este método, el precio medio es igual al producto de cuatro componentes: (1) un factor $T$ que incluye la tendencia de largo plazo de una serie de tiempo; (2) un factor $C$ que representa las variaciones cíclicas alrededor de la tendencia, y que en algunos casos reflejan las oscilaciones suaves que contiene una serie de tiempo debido a la actividad económica y cuya periodicidad fluctúa entre 2 y 5 años; (3) un factor $E$ que representa las variaciones estacionales, las cuales corresponden a fluctuaciones interanuales que se repiten regularmente año a año; y (4) un factor $I$ que representa la componente irregular 
(oscilaciones no sistemáticas), la cual mide las variaciones aleatorias de una serie de tiempo después de eliminar las otras componentes. Lo anterior se puede expresar como

$P_{i j}=P_{t}=T_{t} * C_{t} * E_{t} * I_{t}$, donde los subíndices $i, j$ corresponden al año y mes, respectivamente, y el subíndice $t$ se refiere al valor en el periodo $t$, considerando el carácter discreto de la serie de datos. Se siguió el siguiente procedimiento para cada serie de datos:

Paso 1: Obtención del total móvil de 12 meses. Se sumaron los doce primeros precios estimados a productor (enero a diciembre de 1998), obteniéndose el primer total móvil de 12 meses; este primer total móvil fue centrado el $1^{\circ}$ de julio de 1998. Para obtener segundo total móvil de 12 meses se excluyó el precio medio de enero de 1998 y se incluyó el de enero de 1999; este segundo total móvil fue centrado el $1^{\circ}$ de agosto de 1998 . Este procedimiento se continuó hasta obtener el total móvil de 12 meses centrado el $1^{\circ}$ de julio de 2005.

Paso 2: Obtención del total móvil de 2 años. De los datos obtenidos del Paso 1 se sumaron los dos primeros total móvil centrados el $1^{\circ}$ de julio y $1^{\circ}$ de agosto de 1998 , respectivamente, obteniéndose el primer total móvil de 2 años con datos de 24 meses; este primer total móvil de 2 años fue centrado el $1^{\circ}$ de julio de 1998. Para obtener segundo total móvil de 2 años se excluyó el primer total móvil centrado el $1^{\circ}$ de julio de 1998 y se incluyó el tercer total móvil centrado el $1^{\circ}$ de septiembre de 1998; este segundo total móvil fue centrado el $1^{\circ}$ de agosto de 1998. Este procedimiento se continuó hasta obtener el total móvil de 12 meses centrado el $1^{\circ}$ de junio de 2005.

Paso 3: Obtención del promedio móvil de 2 años centrado en doce meses. Se dividió el total móvil de 2 años obtenido del Paso 2 entre 24 para obtener el promedio de 12 meses, centrados igual que en el Paso 2.

Paso 4: Obtención del Índice Estacional (IE). Se calculó el índice estacional (IE) para cada mes y año dividiendo el precio medio recibido por los productores entre el promedio centrado de 12 meses obtenido del Paso 3; este resultado fue multiplicado por 100 para convertir la razón en un número índice.

Paso 5: Obtención delÍndice EstacionalAjustado(IEA). Primeramente se obtuvo la media mensual modificada (MMM) mediante el promedio simple de los índices estacionales para cada mes (Paso 4); previamente se eliminó el valor máximo y mínimo de cada mes con el objeto de eliminar cualquier distorsión de los valores extremos. Para obtener el valor de la MMM de enero se sumaron los índices estacionales de todos los meses de enero del período 1998 a 2005, excluyendo los valores máximo y mínimo. Para obtener el valor de la MMM de febrero se sumaron los índices estacionales de todos los meses de febrero del período 1998 a 2005, excluyendo los valores máximo y mínimo. Este procedimiento se continuó hasta obtener la MMM correspondiente a diciembre. Luego se calculó el índice estacional ajustado (IEA) para cada mes dividiendo 1.200 entre la suma de las MMM, y multiplicando el resultado obtenido por la correspondiente MMM de cada mes.

Paso 6: Obtención del Índice de Irregularidad Ajustado (IIA). Se calculó el índice de irregularidad ajustado (IIA) siguiendo el mismo procedimiento de cálculo del índice estacional ajustado (IEA). Sin embargo, previamente se obtuvo el índice irregular (II) para cada mes y año, el cual se calculó como el índice cíclico irregular (ICI) entre el índice de tendencia. El índice cíclico irregular (ICI) se obtuvo como el promedio móvil de 2 años centrado en 12 meses (Paso 3) entre el valor de tendencia pronosticado del precio medio estimado a productor.

Paso 7: Obtención del intervalo de dispersión. Finalmente, se estableció un intervalo de dispersión de dos índices estacionales (límites superiores e inferiores), el cual fue obtenido multiplicando y dividiendo el índice estacional ajustado (IEA) por el índice de irregularidad ajustado (IIA).

\section{RESULTADOS Y DISCUSIÓN}

Los precios medios estimados a productor de kiwis y manzanas frescas chilenas se presentan en la Tabla 1. La evolución de precios medios FOB y a productor de kiwis (Figura 1) indica que los precios medios de kiwis siguen una tendencia relativamente estable, los precios desalineados (outlier) de febrero de 2004 y 2005 se explican porque en esos meses se registraron exportaciones extremadamente bajas y no representativas de las condiciones de cosecha y demanda interna de esta fruta. La evolución de precios medios FOB y a productor de manzanas (Figura 2) muestra una tendencia de largo plazo decreciente, lo cual se explica porque la producción mundial ha estado creciendo más rápidamente que el consumo (USDA, 2006) además del masivo ingreso a los principales mercados mundiales de las manzanas provenientes de China, las cuales constituyen el principal sustituto de las manzanas chilenas (Cerda et al., 2004). La comparación de los precios medios FOB y estimados a productor (Tabla 1 y 2) sugiere que las empresas exportadoras transfieren a los productores un porcentaje de variabilidad mayor que las que ellas enfrentan en los mercados internacionales de manzanas frescas chilenas; lo cual se comprueba al observar el $\mathrm{CV}$ anual estimado para cada serie de precios. Lo anterior se explica por la fórmula de cálculo de los precios medios a productor, la cual incorpora una componente variable (comisión, expresada como porcentaje del precio medio FOB) y una componente fija (tarifas, expresada en US $\$ \mathrm{~kg}^{-1}$ ). Lo mismo se puede concluir al observar los indicadores de volatilidad y variabilidad mensual de las series de precios medios de la Tabla 3. Según Lobos y Muñoz (2004) la comisión de exportación (componente variable) es del $8 \%$ del precio FOB y los principales tipos de tarifas (componente fija) son los siguientes: frío, proceso o packing, ducha bins o DPA, uso de bins, control de calidad, certificación SAG, convenios de control de calidad con la Asociación de Exportadores de Chile, materiales de embalaje, fletes desde la planta al puerto, embarque y estiba. Para los exportadores es muy relevante el "flete desde la planta al puerto", ya que esta variable está sujeta a una alta variabilidad y constituye una importante fuente de riesgo debido a la volatilidad asociada a los precios internacionales del petróleo y al tipo de cambio real. En este último caso es muy relevante la acción del gobierno y del Banco Central de Chile en garantizar un nivel mínimo rentable para el tipo de cambio real a través de la política cambiaria. El 
resto de las tarifas son fácilmente predecibles por los exportadores y conocidas por los productores, además de ser relativamente estables en el tiempo.

El análisis de la componente estacional ajustada (Tabla 4) de los precios medios estimados a productor indica que:

(a) los precios medios de kiwis frescos son más altos que el promedio anual en los meses de marzo y desde julio a diciembre. En los otros meses los precios medios fueron inferiores al promedio anual. Los mayores precios medios se observaron en los meses de diciembre y fueron superiores en un $20,4 \%$ al promedio anual. Por otra parte, los menores precios medios se observaron en los meses de junio y fueron inferiores en un $23,6 \%$ al promedio anual.

(b) los precios medios de manzanas frescas son más altos que el promedio anual en los meses de febrero y desde junio a agosto. En los otros meses los precios medios fueron inferiores al promedio anual. Los mayores precios medios se observaron en los meses de julio y fueron superiores en un 38,4\% al promedio anual. Por otra parte, los menores precios medios se observaron en los meses de enero y fueron inferiores en un $26,4 \%$ al promedio anual.

El análisis de variación estacional y de límites superior e inferior (Tabla 4, y Figura 3 y 4) de los precios medios muestran que:

(a) en el mes de marzo y desde julio a diciembre ocurre cierta estabilidad en los precios de kiwis frescos; la mayor variabilidad se observó en el mes de diciembre, con un IEA de 120,4 y límites de 155,5 (superior) y de 93,2 (inferior).

(b) desde febrero a junio y desde agosto a diciembre ocurre cierta estabilidad en los precios de manzanas frescas; la mayor variabilidad se observó en el mes de julio, con un IEA de 138,4 y límites de 175,5 (superior) y de 109,1 (inferior).

(c) los precios medios más bajos observados desde abril a junio (IEA para kiwis frescos) coinciden con el período de mayores volúmenes exportados (Exports de kiwis frescos), los cuales también están representados por las "barras de cantidades" más altas de exportaciones de kiwis frescos de la Figura 3. Este período coincide con los meses de la cosecha (marzo y abril) de kiwis en Chile y es consistente con el período más breve de almacenamiento debido a la corta vida de esta fruta luego de la cosecha.

(d) los precios medios más bajos observados durante los meses de abril (IEA para manzanas frescas) coinciden con el período del mayor volumen exportado (Exports de manzanas frescas), el cual está representado por la "barra de cantidad" más alta en abril de exportaciones de manzanas frescas de la Figura 4. La cosecha de manzanas en Chile se inicia a principios de febrero (en algunas zonas podría iniciarse la última semana de enero), y se embarcan de inmediato con destino a los mercados externos. El mayor porcentaje es estas exportaciones corresponde a Royal Gala y Granny Smith, variedades que se cosechan en función de la disponibilidad de mercado, debido a la corta vida de postcosecha (no más de tres meses en almacenaje). Este resultado está de acuerdo con el planteamiento de Emden (2004) sobre el período de inicio de la cosecha y almacenamiento de manzanas en Chile.
Cabe destacar que los índices de estacionalidad de los precios medios recibidos por los productores resportados por la literatura (Morgado et al., 2004; Lobos y Muñoz, 2005) también mostraron valores más bajos durante los meses en que se concentra la producción. Por otra parte, la alta volatilidad observada en los precios internacionales de kiwis y manzanas frescas chilenas es consistente con la clasificación de bienes normales e inelásticas al precio-propio para las frutas frescas en general, entre ellas kiwis y manzanas (Lee, 1994; Duval \& Biere, 2002; Agbola, 2003; Stewart et al., 2003), o la misma clasificación específicamente para las manzanas (Cerda et al., 2004; Lobos et al., 2005). Los resultados de este trabajo permiten tomar mejores decisiones de producción y optimizar el proceso de cosecha, de manera a disminuir el riesgo asociado a los precios (González et al., 2002) y a las condiciones que prevalecerán en los mercados de destino al momento de la comercialización (Starleaf, 1982).

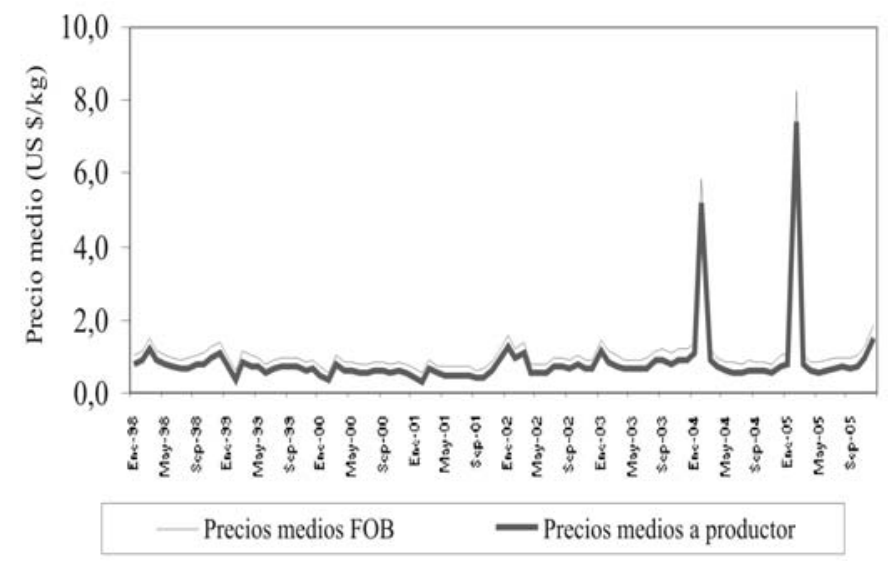

FIGURA 1 - Evolución de precios medios FOB y estimados a productor de kiwis frescos chilenos, 1998-2005.

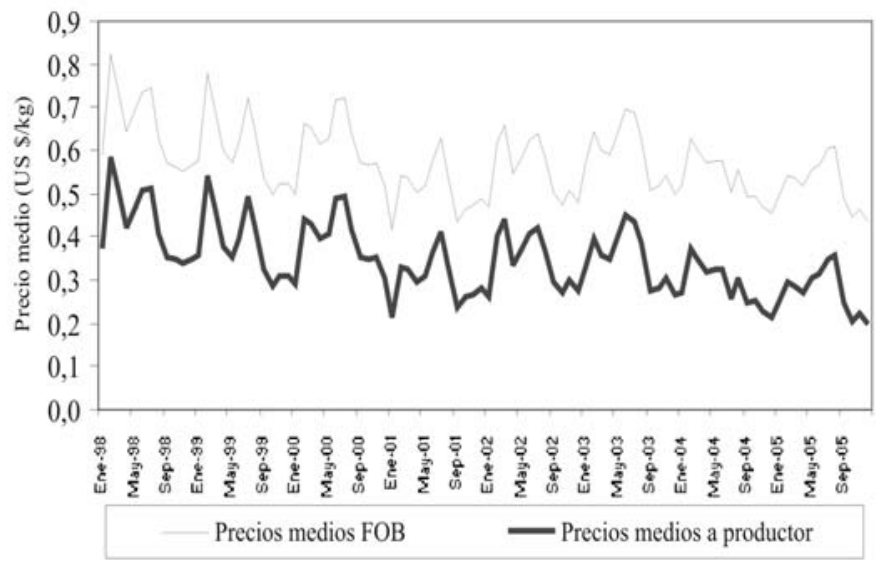

FIGURA 2 - Evolución de precios medios FOB y estimados a productor de manzanas frescas chilenas, 19982005. 


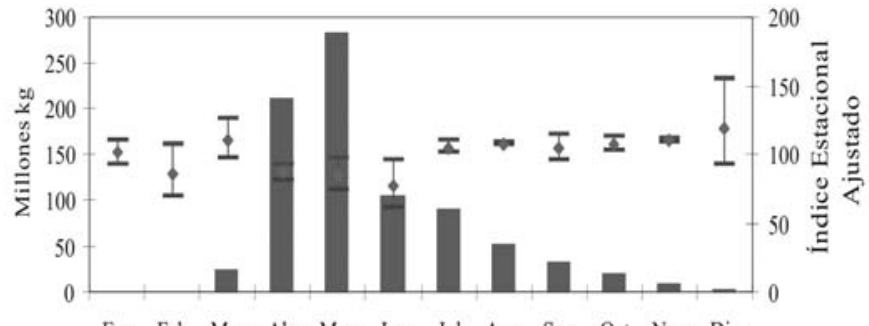

Ene Feb Mar Abr May Jun Jul Ago Sep Oct Nov Dic

-volumen $-\lim$ superior $-\lim$ inferior $\bullet$ IEA

FIGURA 3 - Variación estacional de precios medios a productor de kiwis frescos chilenos, 1998-2005.

("barras de cantidades" volumen de exportaciones de kiwis)

("líneas de precios" representan índice estacional ajustado de precios)

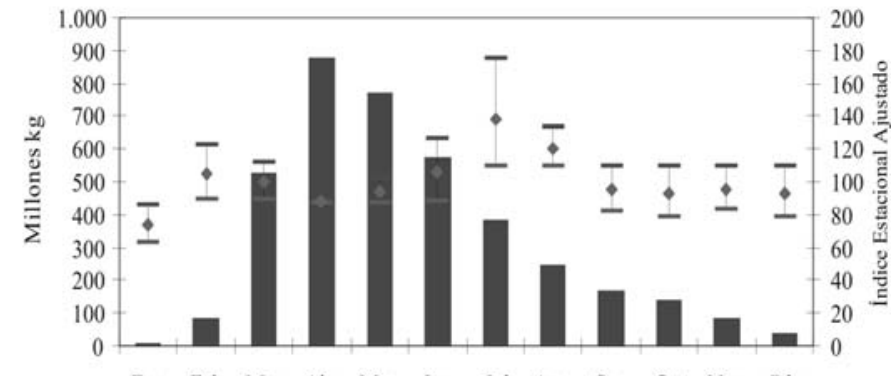

Ene Feb Mar Abr May Jun Jul Ago Sep Oct Nov Dic

-volumen - limsuperior - liminferior \&IEA

FIGURA 4 - Variación estacional de precios medios a productor de manzanas frescas chilenas, 1998-2005

("barras de cantidades" representan volumen de exportaciones de manzanas)

("líneas de precios" representan índice estacional ajustado de precios)
TABLA 1 - Precios medios estimados a productor de kiwis y manzanas frescas chilenas (US\$ kg-1 de diciembre 2005)

\begin{tabular}{|c|c|c|c|c|c|c|c|c|}
\hline \multicolumn{9}{|c|}{ Kiwis frescos } \\
\hline Mes & 1998 & 1999 & 2000 & 2001 & 2002 & 2003 & 2004 & 2005 \\
\hline Ene & 0,76 & 1,09 & 0,50 & 0,43 & 1,26 & 0,63 & 0,86 & 0,80 \\
\hline Feb & 0,89 & 0,37 & 0,50 & 0,44 & 0,94 & 0,62 & 5,16 & 7,40 \\
\hline Mar & 1,21 & 0,86 & 0,75 & 0,65 & 1,09 & 0,72 & 0,90 & 0,76 \\
\hline Abr & 0,87 & 0,75 & 0,61 & 0,52 & 0,57 & 0,66 & 0,70 & 0,59 \\
\hline May & 0,76 & 0,70 & 0,58 & 0,49 & 0,57 & 0,66 & 0,59 & 0,56 \\
\hline Jun & 0,71 & 0,53 & 0,53 & 0,51 & 0,54 & 0,65 & 0,55 & 0,61 \\
\hline Jul & 0,66 & 0,66 & 0,54 & 0,50 & 0,72 & 0,67 & 0,51 & 0,67 \\
\hline Ago & 0,69 & 0,70 & 0,59 & 0,47 & 0,72 & 0,88 & 0,63 & 0,71 \\
\hline Sep & 0,79 & 0,74 & 0,58 & 0,41 & 0,68 & 0,93 & 0,59 & 0,66 \\
\hline Oct & 0,81 & 0,70 & 0,56 & 0,45 & 0,76 & 0,81 & 0,57 & 0,71 \\
\hline Nov & 0,99 & 0,62 & 0,58 & 0,58 & 0,68 & 0,90 & 0,52 & 0,94 \\
\hline Dic & 1,09 & 0,63 & 0,55 & 0,89 & 0,67 & 0,89 & 0,73 & 1,53 \\
\hline Promedio & 0,85 & 0,70 & 0,57 & 0,53 & 0,77 & 0,75 & 1,03 & 1,33 \\
\hline Desvest & 0,17 & 0,17 & 0,07 & 0,13 & 0,22 & 0,12 & 1,31 & 1,93 \\
\hline $\mathrm{CV}(\%)$ & 19,70 & 24,92 & 11,61 & 24,93 & 28,78 & 16,05 & 127,36 & 145,27 \\
\hline \multicolumn{9}{|c|}{ Manzanas frescas } \\
\hline Mes & 1998 & 1999 & 2000 & 2001 & 2002 & 2003 & 2004 & 2005 \\
\hline Ene & 0,37 & 0,36 & 0,29 & 0,21 & 0,26 & 0,33 & 0,27 & 0,25 \\
\hline Feb & 0,59 & 0,54 & 0,44 & 0,33 & 0,40 & 0,40 & 0,37 & 0,29 \\
\hline Mar & 0,51 & 0,47 & 0,43 & 0,33 & 0,44 & 0,36 & 0,35 & 0,29 \\
\hline Abr & 0,42 & 0,38 & 0,39 & 0,30 & 0,34 & 0,35 & 0,32 & 0,27 \\
\hline May & 0,47 & 0,35 & 0,41 & 0,31 & 0,37 & 0,40 & 0,32 & 0,31 \\
\hline Jun & 0,51 & 0,40 & 0,49 & 0,36 & 0,41 & 0,45 & 0,32 & 0,31 \\
\hline Jul & 0,51 & 0,49 & 0,49 & 0,41 & 0,42 & 0,44 & 0,26 & 0,35 \\
\hline Ago & 0,41 & 0,43 & 0,41 & 0,33 & 0,37 & 0,39 & 0,30 & 0,36 \\
\hline Sep & 0,35 & 0,33 & 0,36 & 0,24 & 0,30 & 0,27 & 0,25 & 0,25 \\
\hline Oct & 0,35 & 0,29 & 0,35 & 0,26 & 0,27 & 0,28 & 0,25 & 0,20 \\
\hline Nov & 0,34 & 0,31 & 0,36 & 0,27 & 0,30 & 0,31 & 0,23 & 0,22 \\
\hline Dic & 0,35 & 0,31 & 0,31 & 0,28 & 0,27 & 0,27 & 0,21 & 0,20 \\
\hline Promedio & 0,43 & 0,39 & 0,39 & 0,30 & 0,35 & 0,35 & 0,29 & 0,27 \\
\hline Desvest & 0,08 & 0,08 & 0,07 & 0,05 & 0,06 & 0,06 & 0,05 & 0,05 \\
\hline $\mathrm{CV}(\%)$ & 19,42 & 20,86 & 16,59 & 18,04 & 18,49 & 17,80 & 17,39 & 19,35 \\
\hline
\end{tabular}

Fuente: Datos de la investigación
TABLA 2- Precios medios FOB de kiwis y manzanas frescas chilenas (US\$ kg-1 de diciembre 2005).

\begin{tabular}{|c|c|c|c|c|c|c|c|c|}
\hline \multicolumn{9}{|c|}{ Kiwis frescos } \\
\hline Mes & 1998 & 1999 & 2000 & 2001 & 2002 & 2003 & 2004 & 2005 \\
\hline Ene & 1,01 & 1,37 & 0,73 & 0,65 & 1,55 & 0,90 & 1,16 & 1,10 \\
\hline Feb & 1,16 & 0,59 & 0,73 & 0,66 & 1,21 & 0,88 & 5,84 & 8,27 \\
\hline Mar & 1,50 & 1,12 & 1,01 & 0,88 & 1,36 & 0,99 & 1,20 & 1,05 \\
\hline $\mathrm{Abr}$ & 1,14 & 1,00 & 0,85 & 0,75 & 0,80 & 0,92 & 0,98 & 0,87 \\
\hline May & 1,01 & 0,95 & 0,82 & 0,72 & 0,80 & 0,93 & 0,87 & 0,83 \\
\hline Jun & 0,96 & 0,76 & 0,76 & 0,73 & 0,77 & 0,91 & 0,82 & 0,88 \\
\hline Jul & 0,90 & 0,90 & 0,78 & 0,72 & 0,96 & 0,94 & 0,78 & 0,95 \\
\hline $\mathrm{Ag} 0$ & 0,94 & 0,95 & 0,83 & 0,70 & 0,97 & 1,17 & 0,91 & 0,99 \\
\hline Sep & 1,04 & 0,99 & 0,82 & 0,63 & 0,92 & 1,22 & 0,87 & 0,94 \\
\hline Oct & 1,06 & 0,94 & 0,80 & 0,67 & 1,01 & 1,09 & 0,85 & 1,00 \\
\hline Nov & 1,26 & 0,86 & 0,82 & 0,82 & 0,92 & 1,18 & 0,79 & 1,25 \\
\hline Dic & 1,37 & 0,87 & 0,78 & 1,15 & 0,91 & 1,18 & 1,02 & 1,89 \\
\hline Promedio & 1,11 & 0,94 & 0,81 & 0,76 & 1,02 & 1,03 & 1,34 & 1,67 \\
\hline Desvest & 0,18 & 0,19 & 0,07 & 0,14 & 0,24 & 0,13 & 1,42 & 2,10 \\
\hline CV $(\%)$ & 16,41 & 20,00 & 8,95 & 18,93 & 23,60 & 12,76 & 106,13 & 125,81 \\
\hline \multicolumn{9}{|c|}{ Manzanas frescas } \\
\hline Mes & 1998 & 1999 & 2000 & 2001 & 2002 & 2003 & 2004 & 2005 \\
\hline Ene & 0,59 & 0,58 & 0,50 & 0,41 & 0,47 & 0,57 & 0,52 & 0,49 \\
\hline Feb & 0,82 & 0,78 & 0,67 & 0,54 & 0,62 & 0,64 & 0,63 & 0,54 \\
\hline Mar & 0,74 & 0,69 & 0,65 & 0,54 & 0,66 & 0,60 & 0,60 & 0,54 \\
\hline Abr & 0,64 & 0,60 & 0,62 & 0,50 & 0,55 & 0,59 & 0,57 & 0,52 \\
\hline May & 0,70 & 0,57 & 0,63 & 0,52 & 0,59 & 0,65 & 0,57 & 0,56 \\
\hline Jun & 0,74 & 0,62 & 0,72 & 0,57 & 0,63 & 0,70 & 0,58 & 0,56 \\
\hline Jul & 0,74 & 0,72 & 0,72 & 0,63 & 0,64 & 0,69 & 0,50 & 0,60 \\
\hline Ago & 0,63 & 0,65 & 0,64 & 0,54 & 0,58 & 0,63 & 0,55 & 0,61 \\
\hline Sep & 0,57 & 0,54 & 0,57 & 0,44 & 0,50 & 0,51 & 0,49 & 0,49 \\
\hline Oct & 0,56 & 0,50 & 0,57 & 0,47 & 0,48 & 0,52 & 0,50 & 0,44 \\
\hline Nov & 0,55 & 0,52 & 0,57 & 0,47 & 0,51 & 0,54 & 0,47 & 0,46 \\
\hline Dic & 0,57 & 0,52 & 0,52 & 0,49 & 0,48 & 0,50 & 0,45 & 0,44 \\
\hline Promedio & 0,65 & 0,61 & 0,61 & 0,51 & 0,56 & 0,59 & 0,54 & 0,52 \\
\hline Desvest & 0,09 & 0,09 & 0,07 & 0,06 & 0,07 & 0,07 & 0,05 & 0,06 \\
\hline CV (\%) & 13,90 & 14,47 & 11,56 & 11,60 & 12,44 & 11,51 & 10,15 & 11,06 \\
\hline
\end{tabular}

Fuente: Elaboración propia con datos de ODEPA (2006) 
TABLA 3 - Volatilidad y variabilidad mensual de las series de precios medios (1998-2006) de kiwis y manzanas frescas chilenas

\begin{tabular}{lcccc}
\hline & Kiwis frescos & \multicolumn{3}{c}{ Manzanas frescas } \\
\cline { 2 - 5 } & Precio medio F0B & $\begin{array}{c}\text { Precio medio } \\
\text { a productor }\end{array}$ & Precio medio F0B & $\begin{array}{c}\text { Precio medio } \\
\text { a productor }\end{array}$ \\
\hline Volatilidad ( ,\%) & 41,9 & 47,5 & 11,5 & 17,3 \\
Variabilidad (CV, \%) & 72,8 & 103,6 & 11,9 & 23,7 \\
Promedio (US \$ $\left.\mathrm{gg}^{-1}\right)$ & 1,12 & 0,82 & 1,00 & 0,35 \\
Desvest (US \$ $\mathrm{kg}^{-1}$ ) & 0,81 & 0,84 & 0,12 & 0,08 \\
\hline
\end{tabular}

Fuente: Datos de la investigación

TABLA 4 - Índices estacionales y límites de precios medios recibidos por los productores de kiwis y manzanas frescas chilenas, 1998-2006.

\begin{tabular}{|c|c|c|c|c|c|c|}
\hline \multicolumn{7}{|c|}{ Kiwis frescos } \\
\hline Mes & $\begin{array}{l}\text { Exports } \\
\text { (TM) }\end{array}$ & $\begin{array}{l}\text { Media Mensual } \\
\text { Modificada }\end{array}$ & $\begin{array}{l}\text { Índice Estacional } \\
\text { Ajustado }\end{array}$ & $\begin{array}{l}\text { Índice de Irregularidad } \\
\text { Ajustado }\end{array}$ & $\begin{array}{l}\text { Límite } \\
\text { Superior }\end{array}$ & $\begin{array}{l}\text { Límite } \\
\text { Inferior }\end{array}$ \\
\hline Ene & 607 & 78,9 & 93,5 & 103,4 & 96,7 & 90,4 \\
\hline Feb & 57 & 77,2 & 91,6 & 77,6 & 118,0 & 71,1 \\
\hline Mar & 24.960 & 92,9 & 110,1 & 85,9 & 128,2 & 94,6 \\
\hline Abr & 210.525 & 73,5 & 87,2 & 105,1 & 91,6 & 82,9 \\
\hline May & 282.517 & 71,4 & 84,6 & 115,5 & 97,8 & 73,2 \\
\hline Jun & 103.471 & 64,4 & 76,4 & 123,3 & 94,2 & 61,9 \\
\hline Jul & 89.243 & 89,0 & 105,5 & 104,0 & 109,6 & 101,4 \\
\hline Ago & 52.399 & 91,4 & 108,4 & 97,8 & 110,8 & 106,0 \\
\hline Sep & 33.655 & 88,9 & 105,3 & 109,0 & 114,8 & 96,7 \\
\hline Oct & 19.096 & 89,2 & 105,7 & 103,3 & 109,2 & 102,4 \\
\hline Nov & 8.727 & 94,0 & 111,4 & 97,7 & 114,0 & 108,8 \\
\hline Dic & 2.729 & 101,6 & 120,4 & 77,4 & 155,5 & 93,2 \\
\hline \multicolumn{7}{|c|}{ Manzanas frescas } \\
\hline Mes & $\begin{array}{l}\text { Exports } \\
\text { (TM) }\end{array}$ & $\begin{array}{l}\text { Media Mensual } \\
\text { Modificada }\end{array}$ & $\begin{array}{l}\text { Índice Estacional } \\
\quad \text { Ajustado }\end{array}$ & $\begin{array}{l}\text { Índice de Irregularidad } \\
\text { Ajustado }\end{array}$ & $\begin{array}{l}\text { Límite } \\
\text { Superior }\end{array}$ & $\begin{array}{l}\text { Límite } \\
\text { Inferior }\end{array}$ \\
\hline Ene & 7.863 & 65,8 & 73,6 & 116,7 & 85,8 & 63,0 \\
\hline Feb & 80.474 & 93,7 & 104,8 & 99,7 & 123,3 & 89,1 \\
\hline Mar & 526.529 & 89,1 & 99,6 & 100,6 & 111,5 & 88,9 \\
\hline Abr & 873.394 & 78,9 & 88,2 & 94,3 & 88,9 & 87,4 \\
\hline May & 767.847 & 84,2 & 94,1 & 100,5 & 102,6 & 86,4 \\
\hline Jun & 569.042 & 94,5 & 105,6 & 119,1 & 126,9 & 87,9 \\
\hline Jul & 382.579 & 123,8 & 138,4 & 134,8 & 175,5 & 109,1 \\
\hline Ago & 245.584 & 107,8 & 120,5 & 106,4 & 133,2 & 109,0 \\
\hline Sep & 163.834 & 84,9 & 94,9 & 74,2 & 109,2 & 82,5 \\
\hline Oct & 138.679 & 83,1 & 92,9 & 80,9 & 109,3 & 78,9 \\
\hline Nov & 80.818 & 85,2 & 95,2 & 81,8 & 109,1 & 83,1 \\
\hline Dic & 35.915 & 82,5 & 92,3 & 85,3 & 109,1 & 78,0 \\
\hline
\end{tabular}

Fuente: Datos de la investigación

\section{CONCLUSIONES}

1 - Las empresas exportadoras transmiten a los productores locales una mayor proporción de la volatilidad en los precios medios de kiwis y manzanas frescas chilenas que ellas mismas enfrentan en los mercados internacionales.

2 - La labor desarrollada por los extensionistas agrícolas es fundamental para contribuir al control de gestión y a la planificación de la época de cosecha de los productores.

3 - Desde el punto de vista de los inversionistas en frutales, las decisiones de inversión en plantación de nuevos huertos de kiwis y manzanos, o la ampliación de los existentes, debe considerar el efecto del riesgo asociado a la alta volatilidad de los precios de estas frutas, especialmente si el precio es una variable crítica en la determinación de la rentabilidad esperada.

\section{REFERÊNCIAS}

AGBOLA, F.W. Estimation of food demand patterns in South Africa based on a survey of households. Journal of Agricultural and Applied Economics, Baton Rouge, v.35, n.3, p.663-670, 2003.

BUGUK,C.; HUDSON, D.; HANSON, T. Price volatility spillover in agricultural markets: an examination of U.S. catfish markets. Journal of Agricultural and Resource Economics, Davis, v.28, n.1, p.86-99, 2003.

CERDA, A.; LOBOS, G.; KUFFERATH, E.; SÁNCHEZ, F. Elasticidades de demanda por manzanas chilenas en el mercado de la Unión Europea: una estimación econométrica. Agricultura Técnica, Santiago, v.64, n.4, p.399-408, 2004.

FAO. 2006. Agricultural data. Food and Agriculture Organization of the United Nations. Available at: $<$ http://faostat.fao.org $>$. Accessed: 22 may 2006.

DUVAL, Y.; BIERE, A. Product diffusion and demand for new food products. Agribusiness, San Francisco, v.18, n.1, p.23-36, 2002 .

EMDEN, M.A. Análisis de la industria de la manzana fresca: Situación actual y sus perspectivas. 2004. 96 f. Tesis (doutorado) - Pontificia Universidad Católica de Chile, Santiago, Chile. 2004.

GONZÁLEZ, J.; FRANCISCO, E.; FOSTER, W. Nivel y variabilidad del beneficio económico de rotaciones para la precordillera Andina de la Región del Bío-Bío. Agricultura Técnica, Santiago, v.62, n.3, p.439-449, 2002.

GOODWIN, B.K.; SCHNEPF, R. Determinants of endogenous price risk in corn and wheat markets. The Journal of Futures Markets, Hoboken, v.20, n.8, p.753-774, 2000. 
HANKE, J.E.; REITSCH, A.G. Estadística para negocios. $2^{\text {nd }}$ ed. Madrid: McGraw-Hill, 1997.961p.

HUDSON, D.; COBLE, K. Harvest contract price volatility in cotton. The Journal of Futures Markets, Hoboken, v.19, n.6, p.717-733, 1999.

LEE, J-Y. Fresh fruit consumption in Japan. Agribusiness, San Francisco, v.10, n.6, p.513-520, 1994.

LOBOS, G.; GUTIÉRREZ, L.; PINTO, K. Modelos de demanda por manzanas frescas chilenas en países de la Unión Europea. Revista Brasileira de Fruticultura, Jaboticabal, v.27, n.1, p.143$148,2005$.

LOBOS, G.; MUÑOZ, T. Análisis de los mercados internacionales para las manzanas frescas chilenas, variedad Royal Gala. Revista Frutícola, Chile, v.25, n.3., p.81-88, 2004.

LOBOS, G.; MUÑOZ, T. Índices de estacionalidad de los precios medios recibidos por los productores de manzanas chilenas. Pesquisa Agropecuária Brasileira, Brasília, v.40, n.11, p.10511057,2005 .

MADDALA, G.S. Introducción a la econometría. $2^{\text {nd }}$ ed. México: Prentice-Hall, 1996. 715p.

MENDENHALL, W.; REINMUTH, J.E. Estadística para administración y economía. $3^{\text {rd }}$ ed. México: Iberoamérica, 1981. $707 \mathrm{p}$.

MORGADO, I.F.; AQUINO, C.N.; TERRA, D.C. Economic aspects of the pineapple culture: seasonally of prices in Rio de Janeiro state. Revista Brasileira de Fruticultura, Jaboticabal, v.26, n.1, p.44-47, 2004.
ODEPA. 2006. Base de datos de comercio exterior. Oficina de Estudios y Políticas Agrarias. Disponible en: <http:// www.odepa.cl>. Leído el: 5 mayo 2005.

SEKHAR, C.S.C. Price formation in world wheat markets Implications for policy. Journal of Policy Modeling, New York, v.25, n.1, p.85-106, 2003 a.

SEKHAR, C.S.C. Volatility of agricultural prices: an analysis of mayor international and domestic markets. CRIER, 2003b. 78p. (Working Paper, 103)

STARLEAF, D.R. Macroeconomic policies and their impact upon farm sector. American Journal of Agricultural Economics, Ames, v.64, n.5, p.854-860, 1982.

STEWART, H.; BLISARD, N.; JOLLIFE, D. Do income constraints inhibit spending on fruits and vegetables among low-income households? Journal of Agricultural and Resource Economics, Davis, v.28, n.23, p.465-480, 2003.

TRONCOSO, C.; LOBOS, G. Márgenes de comercialización y concentración industrial en el mercado de frutas y hortalizas en Chile. Agroalimentaria, Mérida, v.18, n.1, p.75-86, 2004.

USDA. United States Department of Agriculture, Foreign Agricultural Services (FAS online). Available at: <http:// www.fas.usda.gov/>. Accessed: 22 may 2006. 